This item was submitted to Loughborough's Research Repository by the author.

Items in Figshare are protected by copyright, with all rights reserved, unless otherwise indicated.

\title{
High-intensity running and energy restriction reduces postprandial lipemia in
} girls

PLEASE CITE THE PUBLISHED VERSION

http://dx.doi.org/10.1249/MSS.0000000000000788

PUBLISHER

(c) American College of Sports Medicine

\section{VERSION}

AM (Accepted Manuscript)

\section{PUBLISHER STATEMENT}

This work is made available according to the conditions of the Creative Commons Attribution-NonCommercialNoDerivatives 4.0 International (CC BY-NC-ND 4.0) licence. Full details of this licence are available at: https://creativecommons.org/licenses/by-nc-nd/4.0/

\section{LICENCE}

CC BY-NC-ND 4.0

\section{REPOSITORY RECORD}

Thackray, Alice, Laura Barrett, and Keith Tolfrey. 2016. "High-intensity Running and Energy Restriction Reduces Postprandial Lipemia in Girls". Loughborough University. https://hdl. handle.net/2134/19428. 


\section{High-intensity running and energy restriction reduces postprandial lipemia in girls}

2 Alice E Thackray ${ }^{1}$, Laura A Barrett ${ }^{1}$ and Keith Tolfrey ${ }^{1}$

$3{ }^{1}$ Paediatric Exercise Physiology Research Group, School of Sport, Exercise and Health

4 Sciences, Loughborough University, Loughborough, LE11 3TU, UK

5

\section{Corresponding author:}

7 Dr Keith Tolfrey

8 School of Sport, Exercise and Health Sciences

$9 \quad$ Loughborough University

10 Loughborough

11 LE11 3TU

12 UK

13 Phone: $+44(0) 1509226355$

14 Fax: $+44(0) 1509226301$

15 Email: K.Tolfrey@lboro.ac.uk.

17 Running title: Exercise, energy restriction and lipemia 


\section{Abstract}

Purpose: This study examined the potency of combining acute high-intensity exercise and energy-intake restriction on postprandial triacylglycerol concentrations ([TAG]) in healthy girls. Methods: Sixteen 11- to 13-year-old girls (mean(SD): body mass 45.1(7.6) kg; peak oxygen uptake $\left(\dot{\mathrm{V}}{ }_{2}\right)$ 43(6) $\mathrm{mL} \cdot \mathrm{kg}^{-1} \cdot \mathrm{min}^{-1}$ ) completed three, 2-day conditions in a counterbalanced, crossover design separated by 14 days. On day 1, participants completed $10 \times 1$ min interval runs (HIIR), $5 \times 1$ min interval runs combined with $0.82(0.19)$ MJ energyintake restriction (HIIR-ER) or rested (CON). Exercise was completed at $100 \%$ maximal aerobic speed, determined from an incremental peak $\dot{\mathrm{VO}}_{2}$ test, with 1 min recovery between intervals. On day 2, capillary blood samples were taken in the fasted state and at predetermined intervals throughout the $6.5 \mathrm{~h}$ postprandial period. A standardised breakfast and lunch were consumed immediately and $4 \mathrm{~h}$, respectively, after the fasting sample. Results: Based on ratios of the geometric means (95\% confidence intervals (CI) for ratios), fasting [TAG] was $16 \%$ and $8 \%$ lower than CON in HIIR $(-24$ to $-7 \%$, effect size $(\mathrm{ES})=0.49, P=$ $0.002)$ and HIIR-ER (-17 to $1 \%, \mathrm{ES}=0.24, P=0.09)$ respectively; HIIR was $8 \%$ lower than HIIR-ER $(-17$ to $1 \%, \mathrm{ES}=0.25, P=0.08)$. The total area under the [TAG] versus time curve was $10 \%$ and $9 \%$ lower than CON in HIIR $(-16$ to $-3 \%$, ES $=0.30, P=0.01)$ and HIIR-ER $(-15$ to $-2 \%$, ES $=0.28, P=0.01)$ respectively; HIIR-ER and HIIR were similar $(-1 \%$; -8 to $6 \%, P=0.80)$. Conclusion: Manipulations of HIIR and ER reduce postprandial [TAG] in girls. The magnitude of effect was marginally, though not meaningfully, greater following HIIR than HIIR-ER.

Keywords: cardiovascular disease risk, energy deficit, exercise intensity, triacylglycerol, young people 
42 Elevated postprandial plasma triacylglycerol concentrations ([TAG]) are implicated in 43 atherogenic development and progression (41), and are established as an independent predictor of cardiovascular disease incidence in women (2). Although the clinical manifestations of atherosclerotic disease are not apparent until adulthood typically, the process of atherosclerosis originates in childhood and progresses over the lifespan (26). The majority of waking hours are postprandial resulting in extended periods of elevated postprandial [TAG]. Therefore, interventions that reduce postprandial [TAG] and delay precursors of atherosclerotic disease should be initiated early in life (26).

Adult studies have shown consistently that acute aerobic exercise (30 min to $3 \mathrm{~h}$ in duration) performed the day before a standardised meal reduces postprandial [TAG] (25), and increases resting whole-body fat oxidation $(9,38)$. Similar reductions in postprandial [TAG] have been reported following acute moderate- to vigorous-intensity exercise in young people (37). Several recent studies in adults highlight the potential efficacy of acute, intermittent highintensity exercise to elicit reductions in postprandial [TAG] (e.g., 13, 38), in addition to improvements in insulin sensitivity and resting whole-body fat oxidation $(38,40)$. Similarly, reductions in postprandial [TAG] have been demonstrated in healthy boys following acute high-intensity interval running (HIIR) (33) and repeated maximal cycle sprints (28). Approximately $80 \%$ of young people in England (19) and globally (17) fail to meet the current international guidelines of $60 \mathrm{~min}$ of daily moderate- to vigorous-intensity exercise for health promotion. Nevertheless, young people typically spend more of their active time engaged in high-intensity activities compared with adults (20). Considering lack of time and enjoyment are frequently highlighted as barriers to exercise participation in adolescent girls (5), the effect of different strategies that reduce the total exercise commitment and promote enjoyment on metabolic health markers should be investigated in girls. Therefore, the first 
aim of the present study was to examine the effect of a single session of HIIR on postprandial plasma [TAG] and resting whole-body fat oxidation in healthy girls.

A small number of studies have compared manipulations in exercise and dietary intake on postprandial [TAG] to determine whether the exercise-evoked reduction in postprandial [TAG] is a consequence of the associated energy deficit or skeletal muscle contraction per se. Acute moderate-intensity exercise appears more efficacious in reducing postprandial [TAG] than isoenergetic mild energy-intake restriction in healthy 11 to 13 year old girls (34) and pre- and post-menopausal women $(15,24)$. Although the combination of moderate-intensity exercise and energy-intake restriction did not exceed the reduction seen for exercise alone in healthy pre-menopausal women, it did at least match it (24). To the author's knowledge, however, no study has examined whether combining exercise with energy-intake restriction to augment the total energy deficit reduces postprandial [TAG] in young people. Therefore, the second aim of the present study was to compare the effect of a smaller dose of HIIR combined with energy-intake restriction (HIIR-ER) with the full HIIR protocol (undertaken previously in boys; 33) and a rest control condition on postprandial plasma [TAG] and whole-body fat oxidation in healthy, recreationally active girls.

\section{Methods}

\section{Participants}

A total of 19 recreationally active girls recruited from local schools volunteered to participate in this study, with results presented for 16 girls (age 12.1(0.7) years; body mass 45.1(7.6) kg; body mass index $18.7(2.1) \mathrm{kg} \cdot \mathrm{m}^{-2}$; peak oxygen uptake $\left.\left(\dot{\mathrm{V} O}{ }_{2}\right) 43(6) \mathrm{mL} \cdot \mathrm{kg}^{-1} \cdot \mathrm{min}^{-1}\right)$ as one girl did not adhere to the required dietary replication and two girls dropped out for personal reasons unrelated to the study. The study procedures were approved by the University Ethical Advisory Committee. Written assent from participants and written informed consent from a 
parent or guardian was obtained before the study commenced. All participants indicated that they were in good general health, had no history of medical conditions that may compromise participation in the study and were not taking any medications or dietary supplements known to influence lipid or carbohydrate metabolism.

Anthropometry and physical maturation

Stature was measured to the nearest $0.01 \mathrm{~m}$ using a fixed stadiometer (Holtain Ltd, Crosswell, UK), body mass was quantified to the nearest $0.1 \mathrm{~kg}$ using a digital scale (Seca 770, Seca Ltd, Hamburg, Germany) and body mass index was calculated as body mass (kg) divided by stature (m) squared. Skinfold thickness was measured at the triceps and subscapular to the nearest $0.2 \mathrm{~mm}$ using Harpenden callipers (Baty International, West

100 Sussex, UK). All measurements were taken on the right-hand side of the body by the same investigator, and the median of three measurements at each site was used to estimate percent body fat (30).

Participants were asked to provide a self-assessment of their level of physical maturity using drawings depicting the five stages of breast and pubic hair development, ranging from 1 indicating pre-pubescence to 5 indicating full sexual maturity (32). Participants identified the stage most closely resembling their current level of sexual development. The median

107 (interquartile range) stage of breast development was 3(2) (stage 1: $n=1$; stage 2: $n=5$; stage 3: $n=5$; stage 4: $n=5$ ) and pubic hair development was 2(3) (stage 1: $n=4$; stage 2: $n$

$109=6$; stage 3: $n=1$; stage 4: $n=3$; stage 5: $n=2$ ).

\section{Preliminary exercise measurements}

111 Participants were familiarised with walking and running on the treadmill (h/p/cosmos

112 mercury med, Nussdorf-Traunstein, Germany) prior to completing an incremental speed- 
113 based treadmill protocol to determine peak $\mathrm{V}_{2}$ and maximal aerobic speed (MAS). The

114 protocol started at $5.0 \mathrm{~km} \cdot \mathrm{h}^{-1}$ with $0.5 \mathrm{~km} \cdot \mathrm{h}^{-1}$ increments every $30 \mathrm{~s}$ until volitional

115 exhaustion, with the treadmill gradient set at 1\%. Heart rate was recorded using short-range

116 telemetry (Polar PE 4000, Kempele, Finland), ratings of perceived exertion were recorded in

117 the last $10 \mathrm{~s}$ of each $30 \mathrm{~s}$ stage, and expired air samples were monitored continuously using

118 an online breath-by-breath gas analysis system (Metalyzer 3B, Cortex, Leipzig, Germany).

119 The analyser was calibrated according to the manufacturer's instructions before the exercise

120 protocol began. Attainment of maximal effort was confirmed based on the presence of a

121 plateau in $\dot{\mathrm{VO}} \mathrm{O}_{2}\left(\leq 3 \%\right.$ with an increase in treadmill speed). In the absence of a plateau in $\dot{\mathrm{V}} \mathrm{O}_{2}$

122 (10 (63\%) participants), an exhaustive effort was confirmed based on the following

123 secondary criteria: a peak heart rate $\geq 95 \%$ of age-predicted maximum (220-chronological

124 age); a respiratory exchange ratio $\geq 1.00$; and clear subjective signs of fatigue. An average of

125 the breath-by-breath $\dot{\mathrm{V}} \mathrm{O}_{2}$ data was taken every 10 s, and peak $\dot{\mathrm{V}} \mathrm{O}_{2}$ was defined as the highest

12630 s rolling average; the treadmill speed corresponding to peak $\dot{\mathrm{V}} \mathrm{O}_{2}$ was recorded as MAS.

\section{Experimental design}

128 Using a within measures, incomplete counterbalanced, crossover design, participants completed three, 2-day experimental conditions separated by a standardised period of 14

130 days: high-intensity interval running (HIIR), high-intensity interval running and energy-

131 intake restriction (HIIR-ER) and rest control (CON). The study design is presented

132 schematically in Figure 1.

Day 1: Intervention day

134 Participants reported to the laboratory at 15:30 and completed all measures by 17:30. Body

135 mass was quantified upon arrival to standardise the meals provided on day 2 (described

136 below). During HIIR and HIIR-ER, the girls completed a 5 min warm-up at 60\% MAS 
137 followed immediately by the acute high-intensity running intervals. The high-intensity

138 running comprised either 10 (HIIR) or 5 (HIIR-ER) $\times 1$ min treadmill runs at $100 \%$ MAS,

139 with 1 min active recovery between each interval. Participants dismounted the treadmill

140 during the active recovery periods and were encouraged to pace around the lab to avoid

141 venous pooling and feeling light headed. Heart rate was monitored continuously and the

142 participants provided a rating of perceived exertion (RPE) in the last $10 \mathrm{~s}$ of each running

143 interval as described previously, and affective valence was quantified at the end of each

144 running interval using a validated feeling scale (FS) (18). Within 5 min of exercise

145 completion, participants completed the modified Physical Activity Enjoyment Scale (PACES;

146 27), and total enjoyment was calculated by summing the 16 responses after eight items were

147 reverse scored. During CON, participants rested in the laboratory for the duration of the visit.

148 Participants maintained and replicated their habitual dietary intake throughout the day in all

149 three conditions, but with a controlled reduction in habitual food energy intake at the evening

150 meal in HIIR-ER by 0.82(0.19) MJ (195(46) kcal).

151 Standardisation of dietary intake and physical activity

152 Participants weighed, recorded and replicated their habitual dietary intake during the $48 \mathrm{~h}$

153 period (pre-intervention and intervention day) before day 2 of the first condition. The girls

154 replicated this diet before the subsequent conditions, but with a controlled reduction in energy

155 intake on the intervention day of HIIR-ER. Participants completing HIIR-ER as the first

156 condition were asked to record their usual dietary intake for two consecutive days at least one

157 week in advance so that the prescribed energy-intake restriction could be calculated and

158 standardised. Two-day diet records were analysed using dietary analysis software (CompEat

159 Pro Version 5.8.0, Nutrition Systems, Banbury, UK). 
160 Participants consumed a cereal snack bar at 19:45 on the intervention day to standardise the 161 overnight fasting period which provided $1.1 \mathrm{~g}$ fat, $15.7 \mathrm{~g}$ carbohydrate, $1.0 \mathrm{~g}$ protein and 337

162 kJ energy. Participants were allowed to drink plain water, but no other drinks or food, before 163 arriving at the laboratory on day 2.

164 An ActiGraph GT1M accelerometer (ActiGraph, Pensacola, Florida, USA) was worn on the 165 pre-intervention and intervention day of each condition, and participants were asked to 166 minimise and replicate their physical activity during this period. The accelerometer was worn 167 on the right hip during waking hours (removed for water-based activities). During data 168 processing, $5 \mathrm{~s}$ epoch data were re-integrated to $60 \mathrm{~s}$ epochs, $60 \mathrm{~min}$ of consecutive zeros, 169 allowing for 2 min of non-zero interruptions was used to remove non-wear, and a minimum 170 of $9 \mathrm{~h}$ of valid wear time was required for a valid day. Physical activity was expressed as 171 average counts per minute (CPM), and intensity cut-points for 12 year olds were applied (39): 172 sedentary $\left(<100\right.$ counts $\left.\cdot \mathrm{min}^{-1}\right)$, light $\left(100-1262\right.$ counts $\left.\cdot \mathrm{min}^{-1}\right)$, moderate $(1262-4136$ 173 counts $\left.\cdot \min ^{-1}\right)$ and vigorous $\left(>4136\right.$ counts $\left.\cdot \min ^{-1}\right)$ activities.

174 Day 2: Postprandial day

175 Following a 12 h overnight fast, participants arrived at the laboratory at 07:45 and provided 176 a fasting capillary blood sample after 10 min seated rest. A standardised breakfast meal was 177 consumed within 15 minutes marking the start of the postprandial period (08:00) (Figure 1). 178 Breakfast consisted of croissants, chocolate spread, whole milk, double cream and milkshake 179 powder. The meal quantity was prescribed relative to body mass and provided $1.5 \mathrm{~g}$ fat 180 (61.3\% of meal total energy), $1.8 \mathrm{~g}$ carbohydrate (32.3\%), 0.4 g protein (6.4\%) and $94 \mathrm{~kJ}$ 181 energy per kilogram body mass. Subsequent capillary blood samples were taken at $0.5,1,3$, 182 4.5, 5 and $6.5 \mathrm{~h}$ following the start of the breakfast, and participants consumed a standardised 183 lunch, within 20 min, at 4 h (Figure 1). Lunch consisted of white bread, butter, mild cheddar 
cheese, potato crisps, whole milk and milkshake powder, and provided $1.3 \mathrm{~g}$ fat (53.5\%), 1.9 g carbohydrate (35.5\%), 0.6 g protein (11.0\%) and 92 kJ energy per kilogram body mass. To ensure consistency across participants and experimental conditions, participants consumed either chocolate or strawberry flavour milkshake powder on all visits. Participants rested throughout the day and were able to read, watch DVD films and play non-active computer games. Participants consumed water ad libitum in the postprandial period of the first condition; the ingested volume was replicated in the subsequent conditions.

191 Resting expired air samples were collected in the semi-supine position for 5 min after each

192 blood sample into 100 L Douglas bags (Cranlea and Company, Birmingham, UK). Oxygen uptake and carbon dioxide production were analysed using a paramagnetic oxygen analyser and an infrared carbon dioxide analyser (Servomex 1400, East Sussex, UK), and the volume of expired air was quantified using a dry gas meter (Harvard Apparatus Ltd, Kent, UK). For each sample, $\dot{\mathrm{VO}}_{2}$, expired carbon dioxide and respiratory exchange ratio were determined, and energy expenditure (EE) and the oxidation of fat and carbohydrate were estimated via indirect calorimetry (12) assuming that the urinary nitrogen excretion rate was negligible. The postprandial expired air data for one girl were spurious so results are presented for 15 200 girls.

\section{Analytical methods}

202 After the hand was pre-warmed for $5 \mathrm{~min}$ in water heated to $40^{\circ} \mathrm{C}$, the fingertip was pierced 203 (Unistik 3 Extra, Owen Mumford, Oxford, UK) and $600 \mu \mathrm{L}$ whole capillary blood was 204 collected into potassium EDTA coated Microvette CB 300 tubes (Sarstedt Ltd, Leicester, 205 UK). The whole blood samples were centrifuged immediately at 12,800 g for 15 min 206 (Eppendorf 5415c, Hamburg, Germany) and the resulting plasma was stored at $-80^{\circ} \mathrm{C}$ for up 207 to two months before subsequent analyses. Plasma [TAG], glucose concentration ([glucose]) 
208 (HORIBA ABX Diagnostics, Montpellier, France) and non-esterified fatty acid 209 concentrations ([NEFA]) (Randox Laboratories Ltd, County Antrim, UK) were analysed by 210 enzymatic, colorimetric methods using a benchtop analyser (Pentra 400, HORIBA ABX 211 Diagnostics, Montpellier, France). The within-batch coefficient of variation for [TAG],

212 [NEFA] and [glucose] were 1.6, 1.5 and 0.8\% respectively. Haemoglobin concentration and 213 haematocrit were also quantified in duplicate in the fasting and final postprandial samples to 214 estimate the acute change in plasma volume (10). Haemoglobin concentration was assessed 215 using the cyanmethemoglobin method; $20 \mu \mathrm{L}$ whole blood was added to $5 \mathrm{~mL}$ Drabkin's 216 solution and the absorbance was quantified photometrically at a wavelength of $546 \mathrm{~nm}$ (Cecil

217 CE1011, Cecil instruments, Cambridge, UK). Haematocrit was quantified using a 218 microhaematocrit centrifuge and reader (Haematospin 1300 Microcentrifuge, Hawksley and 219 Sons Ltd, Sussex, UK).

Statistical analyses

221 Data were analysed using the IBM SPSS Statistics Software for Windows version 21 (IBM

222 Corporation, New York, USA). The trapezium rule was used to calculate the total area under 223 the variable versus time curve for TAG (TAUC-TAG), NEFA (TAUC-NEFA), glucose 224 (TAUC-glucose) and postprandial whole-body EE and substrate oxidation. The TAUC values 225 for substrate oxidation were divided by the total duration of the postprandial period (6.5 h). The incremental area under the plasma concentration versus time curve for TAG (iAUCTAG), NEFA (iAUC-NEFA) and glucose (iAUC-glucose) was calculated using the same 228 method after adjusting for fasting concentrations. The iAUC-NEFA is negative due to the 229 decrease in postprandial [NEFA] from the fasting concentration.

230 Normality of the data was checked using Shapiro Wilk tests. Normally distributed data are 231 presented as mean (SD). Data for free-living physical activity and sedentary time, and 
concentrations of plasma TAG, NEFA and glucose were not normally distributed and were natural log transformed prior to analysis. These data are presented as geometric mean (95\% confidence intervals (CI)) and analysis is based on the ratios of geometric means and 95\% CI for ratios. Homogeneity of variances was confirmed by Mauchly's test of sphericity, and a

236 Greenhouse Geisser correction was applied to the degrees of freedom if the sphericity assumption was violated.

Linear mixed models repeated for condition and interval were used to examine differences between HIIR and HIIR-ER exercise responses for running intervals 1 to 5, and temporal changes between the first and final running interval were modelled with running interval as the sole factor. Dietary intake, free living physical activity and sedentary time, resting wholebody EE and substrate oxidation, fasting concentrations and TAUC and iAUC responses were analysed using separate linear mixed models with condition analysed as a repeated measures factor in the model. Differences in postprandial [TAG], [NEFA] and [glucose] were examined using linear mixed models repeated for condition and time. Temporal changes in TAUC-TAG between experimental conditions were examined over sub-sections of the postprandial period ( 0 to $1 \mathrm{~h}, 1$ to $4.5 \mathrm{~h}$ and 4.5 to $6.5 \mathrm{~h}$ ) using separate linear mixed models with condition as the sole factor. All linear mixed models included a random effect for each participant and were adjusted appropriately for the period effect (29).

Bivariate correlations identifying possible determinants of the exercise-induced changes in

251 TAUC-TAG were quantified using Pearson's product moment correlations. Statistical 252 significance was accepted as $P<0.05$ and absolute standardised effect sizes (ES) are 253 included to supplement important findings. In the absence of a clinical anchor, an ES of 0.2 254 was considered the minimum important difference in all outcome measures, 0.5 moderate and 255 0.8 large (6). 


\section{Dietary intake}

258 Energy and macronutrient intakes were similar on the pre-intervention day across the three conditions $(P \geq 0.14)$. Average daily energy intake was 7.0(1.8) MJ, and dietary intake of

260 protein, carbohydrate and fat was 59.8(19.0) g, 231(70) g and 56.5(14.7) g respectively.

261 Energy and macronutrient intakes during the intervention day are displayed in Table 1.

262 Energy intake on the intervention day of HIIR-ER was lower compared with CON (effect 263 size $($ ES $)=0.60, P<0.001)$ and HIIR $($ ES $=0.54, P<0.001)$; HIIR was significantly, but not meaningfully, lower than CON (ES = 0.06, $P=0.05)$. Absolute protein, carbohydrate and fat intake were lower in HIIR-ER compared with CON and HIIR (ES $=0.35$ to $0.63, P<0.001$ ), but were not different between HIIR and CON ( $P \geq 0.09)$. The only statistical difference in the contribution of protein, carbohydrate and fat to total energy intake was a marginally lower contribution of carbohydrate in HIIR than HIIR-ER (ES $=0.31, P=0.02$ ), and a marginally lower contribution of fat in HIIR-ER than CON $(\mathrm{ES}=0.21, P=0.03)$ and HIIR $(\mathrm{ES}=0.23, P$ $270=0.02)$.

\section{Free-living physical activity and sedentary time}

272 On the pre-intervention day, no differences were seen in physical activity levels or sedentary time across the conditions ( $P \geq 0.27$ ). Physical activity levels and sedentary time on the intervention day are displayed in Table 2. No significant differences were seen across the conditions for daily wear time $(P=0.30)$, sedentary time $(P=0.47)$ or time spent in lightintensity activities $(P=0.15)$. Average counts per minute $(\mathrm{CPM})$ were higher than CON by

277128 counts $\cdot \min ^{-1}$ in HIIR (ES $\left.=1.49, P<0.001\right)$ and by 54 counts $\cdot \min ^{-1}$ in HIIR-ER $(\mathrm{ES}=$ 0.69, $P=0.01)$; HIIR was 74 counts $\cdot \mathrm{min}^{-1}$ higher than HIIR-ER $(\mathrm{ES}=0.80, P=0.005)$. Time spent in moderate-intensity activities was higher in HIIR by 18 min and 15 min compared 
with CON $(\mathrm{ES}=1.06, P=0.001)$ and HIIR-ER $(\mathrm{ES}=0.85, P=0.01)$ respectively; CON and HIIR-ER were similar ( $3 \mathrm{~min} ; P=0.43$ ). Time spent in vigorous-intensity activities was higher than CON by 12 min in HIIR (ES $=1.59, P<0.001)$ and by 7 min in HIIR-ER (ES = 283 1.21, $P<0.001)$; HIIR and HIIR-ER were similar $(P=0.10)$. No differences were observed in free-living physical activity or sedentary time when accounting for the time spent resting or exercising in the laboratory on the intervention day $(P \geq 0.13)$.

Responses to high-intensity interval running (HIIR)

287 The interval running session was performed at an average MAS of $11.5(1.1) \mathrm{km} \cdot \mathrm{h}^{-1}$ and was 288 well tolerated by participants in HIIR and HIIR-ER. Linear mixed models revealed no differences between HIIR-ER and HIIR over running intervals 1 to 5 for heart rate, RPE or FS response $(P \geq 0.11)$. During HIIR, there was a progressive increase from interval 1 to interval 10 for RPE (10(3) to 18(2) respectively; 95\% CI 6 to 10, ES $=2.82, P<0.001$ ) and end interval heart rate $\left(185(12)\right.$ to $202(7)$ beats $\cdot \min ^{-1}$ respectively; 95\% CI 12 to 21 beats $\left.\cdot \mathrm{min}^{-1}, \mathrm{ES}=1.36, P<0.001\right)$, corresponding to $91(4)$ and $99(2) \%$ of peak heart rate respectively (95\% CI 6 to 10\%, ES $=1.99, P<0.001)$. The FS response declined from interval 1 to interval 10 (3(2) to $-2(3)$ respectively; 95\% CI -6 to -3 , ES $=2.99, P<0.001)$. During HIIR-ER, there was a progressive increase from interval 1 to interval 5 for RPE (10(3) to 15 (3) respectively; 95\% CI 3 to 6 , ES $=1.50, P<0.001)$ and end interval heart rate (184(12) to $196(9)$ beats $\cdot \mathrm{min}^{-1}$ respectively; 95\% CI 8 to 16 beats $\cdot \mathrm{min}^{-1}$, $\mathrm{ES}=0.99, P<$ 0.001), corresponding to $90(4)$ and $96(2) \%$ of peak heart rate respectively (95\% CI 4 to 8\%,

$300 \mathrm{ES}=1.51, P<0.001)$, and a decline in the FS response (3(2) to $-1(2)$ respectively; 95\% CI -5 301 to -2 , ES $=1.57, P<0.001)$. The summed PACES score was similar between HIIR-ER and HIIR (57(9) vs. 56(10) respectively; 95\% CI -6 to 3, $P=0.55)$. 
304 Total resting EE over the $6.5 \mathrm{~h}$ postprandial period was similar across the conditions (HIIR 305 2.3(0.3) MJ, HIIR-ER 2.2(0.3) MJ, CON 2.3(0.3) MJ; $P=0.42)$. The relative contribution of 306 fat oxidation to total resting EE tended to be greater than CON (44(17)\%) in HIIR (53(17)\%; $30795 \%$ CI -1 to $20 \%$, ES $=0.50, P=0.09$ ), but HIIR-ER (51(13)\%) was not significantly different to CON (95\% CI -4 to $18 \%$, ES $=0.39, P=0.18$ ) or HIIR (95\% CI -13 to $9 \%, P=$ 309 0.69). Reciprocally, the relative contribution of carbohydrate oxidation to total resting EE 310 tended to be lower compared with CON (56(17)\%) in HIIR (47(17)\%; 95\% CI -20 to 1\%, ES

$311=0.50, P=0.09$ ), but HIIR-ER (49(13)\%) was not significantly different to CON (95\% CI $312-18$ to $4 \%$, ES $=0.39, P=0.18)$ or HIIR (95\% CI -9 to $13 \%, P=0.69)$.

313 Plasma volume changes and fasting [TAG], [NEFA] and [glucose]

314 Average changes in plasma volume between the fasting and $6.5 \mathrm{~h}$ postprandial samples were 315 not different across the three conditions (HIIR -0.3\%, HIIR-ER 0.4\%, CON $-0.4 \%$; $P=0.77$ ).

316 Therefore, the raw plasma [TAG], [NEFA] and [glucose] were used in all statistical analyses 317 without adjustment. The fasting plasma [TAG], [NEFA] and [glucose] for each condition are 318 displayed in Table 3. Linear mixed models revealed differences across the conditions in 319 fasting plasma [TAG] $(P=0.01)$ and $[\mathrm{NEFA}$ ( $(P=0.04)$, but not [glucose] $(P=0.41)$. 320 Specifically, fasting plasma [TAG] was $16 \%$ and $8 \%$ lower than CON in HIIR (ES $=0.49, P$ $321=0.002)$ and HIIR-ER $(\mathrm{ES}=0.24, P=0.09)$ respectively; HIIR was $8 \%$ lower than HIIR-ER 322 (ES $=0.25, P=0.08)$. Fasting plasma [NEFA] was $22 \%$ and $20 \%$ lower than CON in HIIR $323(\mathrm{ES}=0.65, P=0.02)$ and HIIR-ER $(\mathrm{ES}=0.58, P=0.04)$ respectively; HIIR-ER and HIIR 324 were not significantly different $(-3 \% ; P=0.78)$. 
Plasma TAG responses over the postprandial period for HIIR, HIIR-ER and CON are shown

in Figure 2. Linear mixed models revealed differences in postprandial plasma [TAG] over time across conditions (main effect condition $P<0.001$; main effect time $P<0.001$; condition by time interaction $P=0.71$ ). Mean postprandial plasma [TAG] was $11 \%$ and $8 \%$ lower than CON in HIIR ( -14 to $-7 \%$, ES $=0.27, P<0.001)$ and HIIR-ER $(-12$ to $-4 \%$, ES = $0.21, P<0.001)$ respectively; HIIR-ER and HIIR were similar $(-3 \% ;-7$ to $2 \%, P=0.24)$. The TAUC-TAG was $10 \%$ and $9 \%$ lower than CON in HIIR $(\mathrm{ES}=0.30, P=0.01)$ and HIIR-ER (ES $=0.28, P=0.01)$ respectively; HIIR-ER and HIIR were similar $(-1 \% ; P=0.80)$ (Table 3). Specifically, TAUC-TAG was lower after HIIR than CON between 0 to 1 h by $16 \%$ (-22 to $-9 \%$, ES $=0.53, P<0.001)$ and 1 to 4.5 h by $11 \%(-17$ to $-4 \%$, ES $=0.31, P=0.003)$; HIIR-ER was lower than CON between 0 to 1 h by $11 \%$ (-17 to $-4 \%$, ES $=0.37, P=0.003$ ) and 1 to 4.5 h by $10 \%(-16$ to $-4 \%, \mathrm{ES}=0.30, P=0.005)$. No differences in TAUC-TAG over sub-sections of the total postprandial period were seen between HIIR-ER and HIIR ( $P \geq$ 0.16). No differences were seen in iAUC-TAG across the conditions $(P=0.53)$ (Table 3).

Individual changes (delta) in TAUC-TAG for HIIR and HIIR-ER relative to CON are shown in Figure 3. The reductions in TAUC-TAG following HIIR and HIIR-ER were greater than changes in CON for ten (63\%) and eleven (69\%) girls respectively. Meaningful positive correlations were identified between the intervention-induced change in fasting plasma

344 [TAG] and the change in TAUC-TAG relative to CON for HIIR $(r=0.52, P=0.04)$ and 345 HIIR-ER $(\mathrm{r}=0.59, P=0.02)$. The measured physical and physiological characteristics, 346 dietary intake (Table 1), free-living physical activity and sedentary time (Table 2), exercise responses, resting whole-body EE and substrate oxidation and fasting [NEFA] or [glucose] 
349 HIIR or HIIR-ER. The Pearson's product moment correlation for the individual changes in

350 TAUC-TAG between HIIR and HIIR-ER was small $(\mathrm{r}=0.31, P=0.25)$.

351 No differences were observed in postprandial plasma [NEFA] across the conditions over time

352 (main effect condition $P=0.58$; main effect time $P<0.001$; condition by time interaction $P=$

353 0.57). No meaningful differences were evident for TAUC-NEFA across the conditions $(P=$

354 0.45) (Table 3). The iAUC-NEFA was 56\% and 55\% higher than CON in HIIR (ES $=0.67, P$

$355=0.01)$ and HIIR-ER $(\mathrm{ES}=0.65, P=0.01)$ respectively; HIIR-ER and HIIR were not

356 different (1\%; $P=0.95)$ (Table 3).

357 Linear mixed models revealed a trend for differences in postprandial plasma [glucose] over

358 time (main effect condition $P=0.06$; main effect time $P<0.001$; condition by time

359 interaction $P=0.77)$. The TAUC-glucose was $4 \%$ higher in HIIR compared with CON $(E S=$

$3600.58, P=0.01)$, but HIIR-ER was not significantly different to HIIR $(-1 \% ; P=0.27)$ or CON

361 (2\%; $P=0.08)$ (Table 3). The only significant difference in iAUC-glucose was a greater

362 response in HIIR compared with HIIR-ER (39\%; ES = 1.43, $P=0.04$ ) (Table 3).

\section{Discussion}

364 The primary finding from the present study is that acute manipulations of low volume HIIR and ER completed the day before standardised meals reduced postprandial plasma [TAG] and increased whole-body fat oxidation in healthy, 11 to 13 year old girls. The magnitude of this effect was marginally, although not meaningfully, greater following HIIR than HIIR-ER. The exercise and diet interventions were well tolerated by all participants and, therefore, may have practical metabolic health benefits in similar cohorts.

The exercise and dietary restriction induced reductions in fasting plasma [TAG] support the majority of previous exercise postprandial studies in young people (e.g., 3, 28, 34, 35). 
372 Although the lower fasting plasma [TAG] in HIIR and HIIR-ER are likely to influence the subsequent postprandial TAG response (7), substantial intra-individual variation is evident in childhood fasting [TAG] (36), and fasting [TAG] are less predictive of cardiovascular disease risk than postprandial [TAG] in women (2).

Several adult studies have reported reductions in postprandial [TAG] following a single session of intermittent, high-intensity exercise (e.g., 13, 38); however, this finding is not universal $(1,31)$. The contrasting results in these studies may reflect the variety of highintensity exercise protocols adopted which, coupled with differences in participant characteristics, exercise timing, meal content and blood sampling, is likely to promote heterogeneity in the individual responses $(1,31)$. Nevertheless, we have demonstrated previously that a single session of HIIR promotes moderate reductions in postprandial plasma [TAG] in 11 to 12 year old boys (33). The current study extends this novel finding to 11 to 13 year old girls, and supports the commonly reported reductions in postprandial [TAG] following acute moderate- to vigorous-intensity exercise in boys and girls (37) and repeated maximal cycle sprints in boys (28).

An additional novel feature of the current study was the inclusion of a condition combining a lower volume of HIIR with a small reduction in energy intake (0.82(0.19) MJ, 195(46) kcal), which reduced postprandial plasma [TAG] to a similar extent as the full HIIR protocol ( 10\%; Table 3, Figure 2). Acute energy-intake restriction alone has been shown to elicit a small reduction in postprandial [TAG] previously in healthy girls $(-10 \%$, ES $=0.32 ; 34)$ and pre-menopausal women (-12\%; 24). Although an exercise-induced energy deficit appears a more potent stimulus to reduce postprandial [TAG] than an isoenergetic diet-induced energy deficit in girls (34) and women (15, 24), the combination of light walking and energy-intake restriction did match the reduction seen for exercise alone in sedentary, pre-menopausal women (24). The similar reduction in postprandial plasma [TAG] following HIIR and HIIR- 
397 ER is promising, and highlights the potential for metabolic health benefits following time398 efficient exercise combined with manageable dietary restriction in girls. A combination of 399 low volume, high-intensity exercise and mild dietary energy intake restriction may represent 400 a practical and attractive alternative in girls who struggle to accumulate sufficient physical 401 activity for health. It contributes to providing girls with a variety of lifestyle options that can 402 reduce postprandial plasma [TAG] and may have important long-term metabolic health 403 implications if employed regularly, but further work is required to support this in young 404 people. One limitation of the present study is that the girls recruited were healthy and recreationally active. Therefore, further research is needed in overweight/obese girls who 406 may require appropriate exercise and dietary interventions for weight management and 407 improvements in the lipid profile.

408 The mechanisms underpinning the acute exercise- and diet-induced reductions in postprandial 409 plasma [TAG] in young people were not measured directly in the present study due to the 410 invasive nature of the methods required to do this accurately. In adults, two primary pathways 411 have been proposed involving the increased clearance of circulating TAG facilitated by 412 enhanced lipoprotein lipase (LPL) activity (16) and/or the secretion of fewer, TAG-richer 413 very low-density lipoproteins (VLDL) that have a higher affinity for LPL (23). A recent 414 stable isotope enrichment study in obese women suggested that the TAG-lowering effect of 415 acute exercise is mediated by a reduced abundance of endogenous fatty acids in plasma TAG 416 and not the enhanced clearance of dietary fat (9). The notion that endogenous, and not 417 exogenous, TAG metabolism exerts a stronger influence on the postprandial TAG response is 418 indirectly supported by the current study evidenced by the small differences in iAUC-TAG 419 between the conditions, and the meaningful relationship seen between the intervention420 induced changes in fasting plasma [TAG] and TAUC-TAG for HIIR $(r=0.52, P=0.04)$ and 421 HIIR-ER $(\mathrm{r}=0.59, P=0.02)$. 
422 Although whole-body fat oxidation was not statistically significant between the three 423 conditions, a thorough appraisal of the mean differences and absolute standardised ES 424 revealed that HIIR was 8\% higher than CON (ES = 0.50) and HIIR-ER was 7\% higher than 425 CON (ES = 0.39). Therefore, combinations of HIIR and ER appears to elevate resting whole426 body fat oxidation the following day, which represents a novel finding in young people and 427 supports exercise postprandial studies in adults employing acute high-intensity exercise 428 protocols $(38,40)$. The post-exercise shift in whole-body substrate utilisation towards fat oxidation has been linked to a number of regulatory mechanisms promoting the resynthesis of depleted skeletal muscle and/or hepatic glycogen stores (21). Circulating plasma fatty acids and triacylglycerol-rich lipoproteins (TRL) are potential lipid sources utilised for oxidation, which is in agreement with the lower postprandial plasma [TAG] after HIIR and HIIR-ER, likely mediated by enhanced LPL activity $(16,21)$. However, the similar postprandial NEFA response between the three experimental conditions suggests that plasma fatty acids did not contribute to the greater whole-body fat oxidation in HIIR and HIIR-ER. Nevertheless, it is possible that differences in plasma [NEFA] were evident before the commencement of the postprandial period considering large increases in plasma free fatty acids have been shown in the early post-exercise recovery period (21). The lack of association between whole-body fat oxidation and indices of lipemia in the current study contrasts previous findings in adults (38), suggesting that exercise- and diet-induced changes in postprandial plasma [TAG] and 441 whole-body fat oxidation may occur independently in girls. Nevertheless, elevated 442 postprandial [TAG] are associated independently with cardiovascular disease risk in women 443 (2), and low resting fat oxidation with an increased risk of weight gain (11) and Type 2 444 diabetes mellitus (4), highlighting the potential efficacy of acute high-intensity exercise and dietary restriction to improve metabolic health outcomes early in life. 
446 Although the clinical significance of our findings cannot be established, the majority (93\%)

447 of the postprandial TAG samples were below the $2.3 \mathrm{mmol} \cdot \mathrm{L}^{-1}$ threshold considered a

448 desirable concentration in young people (22). Based on the physical activity data, nine (56\%)

449 girls in the present study were achieving the current international physical activity 450 recommendations, although it should be noted that this is not a valid measure of habitual 451 physical activity as the girls were asked to minimise and replicate their physical activity 452 levels over a short measurement period. The majority of girls in England and globally 453 (approximately 80\%) fall short of the current physical activity guidelines for health $(17,19)$, 454 and time and enjoyment are reported frequently as barriers to exercise participation in 455 adolescent girls (5). Therefore, the potential for HIIR and HIIR-ER, with a total exercise time 456 commitment of 24 and 14 min respectively (including warm-up and active recovery between intervals), to reduce postprandial plasma [TAG] and increase resting whole-body fat oxidation in girls is encouraging. The girls spent a greater amount of time engaged in 459 vigorous-intensity activities in HIIR and HIIR-ER, and a greater amount of time in moderateintensity activities in HIIR on the intervention day as a result of the prescribed exercise intervention. There were no differences between conditions after accounting for the time spent resting or exercising in the laboratory, suggesting that the implemented between condition control of free-living physical activity and sedentary time was effective. The high464 intensity nature of the exercise adopted in the present study may better reflect the activity 465 patterns of young people who spend a greater proportion of time engaged in high-intensity 466 activities than adults (20). Furthermore, it has been demonstrated that children associate 467 moderate-intensity exercise interspersed with short high-intensity efforts with greater 468 perceived enjoyment than completing continuous moderate-intensity exercise alone (8). In 469 the present study, the similarly high PACES score between HIIR and HIIR-ER suggests 
470 interval running performed at a high-intensity may be an attractive exercise model in girls 471 independent of whether five or ten 1 min intervals are completed.

472 Previous high-intensity exercise postprandial studies highlight the substantial heterogeneity 473 evident in postprandial TAG responses in young people (33) and adults $(1,31)$. We have 474 shown previously in boys that exercising at a higher relative exercise intensity during HIIR is associated with a greater reduction in postprandial plasma [TAG] (33); however, this relationship was not apparent in the current study with girls, and the other measured variables in the study could not explain any of the heterogeneity present. A study with adults reported that exercise-induced changes in 3-OHB, a marker of hepatic fatty acid oxidation, was a strong predictor of the moderate-intensity exercise-induced reduction in fasting and postprandial [TAG] (14). Although this marker may explain some of the heterogeneity in the present study, we measured postprandial 3-OHB concentrations but the assay was unable to detect concentrations of 3-OHB in the majority of fasting and postprandial samples; therefore, further investigation is required in young people.

The higher postprandial plasma [glucose] after HIIR compared with CON supports a recent 485 study in girls adopting a moderate-intensity exercise protocol (34); however, the majority of 486 previous exercise postprandial studies in young people report no difference in postprandial [glucose] following acute exercise (e.g., 3, 28). The reason for this discrepant finding is not known; however, it is unlikely that the higher postprandial [glucose] in HIIR is implicated in the TAG-lowering effect of HIIR considering glucose has not been linked to the potential 490 mechanistic pathways discussed above. Nevertheless, all participants in the present study demonstrated a healthy postprandial glucose profile independent of the experimental condition and the time of glucose measurement, suggesting the girls exhibited good glycemic control. 
494 The present study is limited as the exercise protocol comprised running; therefore, the

495 findings may not generalise to other exercise modalities such as cycling and game-based

496 activities. Despite this limitation, the exercise protocol adopted in the present study is

497 attainable for young people to achieve in a natural setting.

498 In conclusion, acute manipulations of low volume HIIR and ER completed the day before 499 standardised meals reduced postprandial plasma [TAG] and increased resting whole-body fat 500 oxidation in healthy, 11 to 13 year old girls. The magnitude of this effect was marginally, 501 though not meaningfully, greater following HIIR than HIIR-ER. Low volume, HIIR 502 performed alone or in combination with a mild reduction in habitual energy intake may represent time-efficient and enjoyable strategies to improve metabolic health in girls, but

504 further work is required to examine this chronically and in overweight/obese girls for whom 505 HIIR-ER may be an efficacious intervention.

\section{Acknowledgements}

507 We thank Woodbrook Vale School and Rawlins Academy in Loughborough for their support 508 and understanding throughout this research. We also thank the participants and their parents 509 for their commitment throughout the study. This study was supported in part by the 510 NASPEM Marco Cabrera Student Research Grant and by funding available internally 511 through Loughborough University.

512 The research was supported by the National Institute for Health Research (NIHR) Diet,

513 Lifestyle \& Physical Activity Biomedical Research Unit based at University Hospitals of

514 Leicester and Loughborough University. The views expressed are those of the authors and 515 not necessarily those of the NHS, the NIHR or the Department of Health. 


\section{Conflict of interest}

517 The authors declare that they have no conflict of interest. The results of the present study do

518 not constitute endorsement by ACSM. 
1. Allen E, Gray P, Kollias-Pearson A, Oag E, Pratt K, Henderson J, Gray SR. The effect of short-duration sprint interval exercise on plasma postprandial triacylglycerol levels in young men. J Sports Sci. 2014;32(10):911-6.

2. Bansal S, Buring JE, Rifai N, Mora S, Sacks FM, Ridker PM. Fasting compared with nonfasting triglycerides and risk of cardiovascular events in women. JAMA. 2007;298(3):309-16.

3. Barrett LA, Morris JG, Stensel DJ, Nevill ME. Exercise and postprandial plasma triacylglycerol concentrations in healthy adolescent boys. Med Sci Sports Exerc.

4. Blaak EE, Wolffenbuttel BHR, Saris WHM, Pelsers MMAL, Wagenmakers AJM. Weight reduction and the impaired plasma-derived free fatty acid oxidation in Type 2 diabetic subjects. J Clin Endocrinol Metab. 2001;86(4):1638-44.

5. Butt J, Weinberg RS, Breckon JD, Claytor RP. Adolescent physical activity participation and motivational determinants across gender, age, and race. $J$ Phys Act Health. 2011;8(8):1074-83.

6. Cohen J. Statistical power analysis for the behavioural sciences. 2nd ed. Hillsdale (NJ):

7. Couch SC, Isasi CR, Karmally W, Blaner WS, Starc TJ, Kaluski D, Deckelbaum RJ,

8. Crisp NA, Fournier PA, Licari MK, Braham R, Guelfi KJ. Adding sprints to continuous exercise at the intensity that maximises fat oxidation: implications for acute energy balance and enjoyment. Metabolism. 2012;61(9):1280-8.

9. Davitt PM, Arent SM, Tuazon MA, Golem DL, Henderson GC. Postprandial triglyceride 546 and free fatty acid metabolism in obese women after either endurance or resistance exercise. J Appl Physiol. 2013;114(12):1743-54. 
10. Dill DB, Costill DL. Calculation of percentage changes in volumes of blood, plasma, and red cells in dehydration. J Appl Physiol. 1974;37(2):247-8.

11. Ellis AC, Hyatt TC, Hunter GR, Gower BA. Respiratory quotient predicts fat mass gain in premenopausal women. Obesity (Silver Spring). 2010;18(12):2255-9.

12. Frayn KN. Calculation of substrate oxidation rates in vivo from gaseous exchange. $J$ Appl Physiol. 1983;55(2):628-34.

13. Gabriel B, Ratkevicius A, Gray P, Frenneaux MP, Gray SR. High-intensity exercise attenuates postprandial lipaemia and markers of oxidative stress. Clin Sci (Lond). 2012;123(5):313-21.

14. Gill JMR, Al-Mamari A, Ferrell WR, Cleland SJ, Perry CG, Sattar N, Packard CJ, Caslake MJ, Petrie JR. Effect of prior moderate exercise on postprandial metabolism in men with type 2 diabetes: heterogeneity of responses. Atherosclerosis. 2007;194(1):13443.

15. Gill JMR, Hardman AE. Postprandial lipemia: effects of exercise and restriction of energy intake compared. Am J Clin Nutr. 2000;71(2):465-71.

16. Gill JMR, Herd SL, Vora V, Hardman AE. Effects of a brisk walk on lipoprotein lipase activity and plasma triglyceride concentrations in the fasted and postprandial states. Eur J Appl Physiol. 2003;89(2):184-90.

17. Hallal PC, Andersen LB, Bull FC, Guthold R, Haskell W, Ekelund U. Global physical activity levels: surveillance progress, pitfalls, and prospects. Lancet. 2012;380(9838):247-57.

18. Hardy CJ, Rejeski WJ. Not what, but how one feels: the measurement of affect during exercise. J Sport Exerc Psychol. 1989;11(3):304-17.

19. Health Survey for England 2012. Volume 1: Health, social care and lifestyles. Chapter 3: Physical activity in children. London: Health and Social Care Information Centre; 2012. p. 8. Available from Health and Social Care Information Centre.

20. Hoos MB, Kuipers H, Gerver WJM, Westerterp KR. Physical activity pattern of children assessed by triaxial accelerometry. Eur J Clin Nutr. 2004;58(10):1425-8. 
575

576

577

578

579

580

581

582

583

584

585

586

587

588

589

590

591

592

593

594

595

596

597

598

599

600

601

602

21. Kiens B, Richter EA. Utilization of skeletal muscle triacylglycerol during postexercise recovery in humans. Am J Physiol. 1998;275(2 Pt 1):E332-7.

22. Kolovou GD, Bilianou H, Mikhailidis DP. Postprandial lipemia in children and adolescents. Curr Vasc Pharmacol. 2011;9(3):318-20.

23. Magkos F, Wright DC, Patterson BW, Mohammed BS, Mittendorfer B. Lipid metabolism response to a single, prolonged bout of endurance exercise in healthy young men. Am J Physiol Endocrinol Metab. 2006;290(2):E355-62.

24. Maraki M, Magkos F, Christodoulou N, Aggelopoulou N, Skenderi KP, Panagiotakos D, Kavouras SA, Sidossis LS. One day of moderate energy deficit reduces fasting and postprandial triacylglycerolemia in women: the role of calorie restriction and exercise. Clin Nutr. 2010;29(4):459-63.

25. Maraki MI, Sidossis LS. The latest on the effect of prior exercise on postprandial lipaemia. Sports Med. 2013;43(6):463-81.

26. McGill HC, McMahan CA, Herderick EE, Malcom GT, Tracy RE, Strong JP. Origin of atherosclerosis in childhood and adolescence. Am J Clin Nutr. 2000;72(5):1307S-15S.

27. Motl RW, Dishman RK, Saunders R, Dowda M, Felton G, Pate RR. Measuring enjoyment of physical activity in adolescent girls. Am J Prev Med. 2001;21(2):110-7.

28. Sedgwick MJ, Morris JG, Nevill ME, Barrett LA. Effect of repeated sprints on postprandial endothelial function and triacylglycerol concentrations in adolescent boys. $J$ Sports Sci. 2015;33(8):806-16.

29. Senn S. Cross-over trials in clinical research. Chichester, UK: Wiley; 1993. pp. 130-8.

30. Slaughter MH, Lohman TG, Boileau RA, Horswill CA, Stillman RJ, Van Loan MD, Bemben DA. Skinfold equations for estimation of body fatness in children and youth. Hum Biol. 1988;60(5):709-23.

31. Tan MS, Mok A, Yap MC, Burns SF. Effect of sprint interval versus continuous cycling on postprandial lipaemia. J Sports Sci. 2013;31(9):989-95.

32. Tanner JM. Growth at adolescence. 2nd ed. Oxford, UK: Blackwell Scientific Publications; 1962. pp. 28-39. 
603

604

605

606

607

608

609

610

611

612

613

614

615

616

617

618

619

620

621

622

623

624

625

33. Thackray AE, Barrett LA, Tolfrey K. Acute high-intensity interval running reduces postprandial lipemia in boys. Med Sci Sports Exerc. 2013;45(7):1277-84.

34. Thackray AE, Barrett LA, Tolfrey K. Acute effects of energy deficit induced by moderate-intensity exercise or energy-intake restriction on postprandial lipemia in healthy girls. Pediatr Exerc Sci. 2015;27(2):192-202.

35. Tolfrey K, Bentley C, Goad M, Varley J, Willis S, Barrett L. Effect of energy expenditure on postprandial triacylglycerol in adolescent boys. Eur J Appl Physiol. 2012;112(1):23-31.

36. Tolfrey K, Campbell IG, Jones AM. Intra-individual variation of plasma lipids and lipoproteins in prepubescent children. Eur J Appl Physiol. 1999;79(5):449-56.

37. Tolfrey K, Thackray AE, Barrett LA. Acute exercise and postprandial lipemia in young people. Pediatr Exerc Sci. 2014;26(2):127-37.

38. Trombold JR, Christmas KM, Machin DR, Kim IY, Coyle EF. Acute high-intensity endurance exercise is more effective than moderate-intensity exercise for attenuation of postprandial triglyceride elevation. J Appl Physiol. 2013;114(6):792-800.

39. Trost SG, Pate RR, Sallis JF, Freedson PS, Taylor WC, Dowda M, Sirard J. Age and gender differences in objectively measured physical activity in youth. Med Sci Sports Exerc. 2002;34(2):350-5.

40. Whyte LJ, Ferguson C, Wilson J, Scott RA, Gill JMR. Effects of single bout of very high-intensity exercise on metabolic health biomarkers in overweight/obese sedentary men. Metabolism. 2013;62(2):212-9.

41. Zilversmit DB. Atherogenesis: a postprandial phenomenon. Circulation. 1979;60(3):473-85. 


\section{Figure legends}

627 Figure 1 Diagram of the 2-day study protocol. TAG, triacylglycerol; NEFA, nonesterified fatty acids. ${ }^{+}$Evening meal replicated from the first condition but with a small reduction in energy intake in HIIR-ER.

630 Figure 2 Fasting (F) and postprandial plasma triacylglycerol concentrations ([TAG]) in the control (CON), high-intensity interval running and energy-intake restriction (HIIR-ER) and high-intensity interval running (HIIR) conditions ( $n=16)$. Values are mean (SD). Black rectangles denote consumption of breakfast and lunch meals at 08:00 and 12:00, respectively. Main effect condition $P<0.001$; main effect time $P<0.001$; condition by time interaction $P=0.71$.

636 Figure 3 Individual changes (delta) in the total area under the plasma triacylglycerol (TAG) concentration versus time curve (TAUC) between the high-intensity interval running (HIIR) and high-intensity interval running and energy-intake restriction (HIIR-ER) conditions compared with the control condition (CON): according to the size of the intervention-induced change in TAUC-TAG; thus, the order of the individual participants is not identical in A and B. A negative CON. 
Table 1 Energy and macronutrient intakes during the intervention day of the high-intensity interval running (HIIR), high-intensity interval running and energy-intake restriction (HIIR-ER) and control (CON) conditions.

\begin{tabular}{|c|c|c|c|c|c|c|}
\hline & HIIR & HIIR-ER & CON & $\begin{array}{c}\text { CON vs. HIIR } \\
95 \% \mathrm{CI}^{*}\end{array}$ & $\begin{array}{c}\text { CON vs. HIIR-ER } \\
95 \% \text { CI* }^{*}\end{array}$ & $\begin{array}{c}\text { HIIR-ER vs. HIIR } \\
95 \% \text { CI* }^{*}\end{array}$ \\
\hline Energy $\left(\mathrm{MJ} \cdot \mathrm{day}^{-1}\right)$ & $6.4(1.4)$ & $5.6(1.5)$ & $6.5(1.4)$ & -0.19 to $0.00^{\mathrm{a}}$ & -0.9 to $-0.8^{\mathrm{b}}$ & 0.7 to $0.9^{\mathrm{c}}$ \\
\hline Protein $\left(g \cdot\right.$ day $\left.^{-1}\right)$ & $54.4(20.0)$ & $47.1(19.2)$ & $54.1(19.8)$ & -1.4 to 1.9 & -8.2 to $-4.9^{b}$ & 5.2 to $8.4^{\mathrm{c}}$ \\
\hline $\mathrm{CHO}\left(\mathrm{g} \cdot \mathrm{day}^{-1}\right)$ & $218(42)$ & $196(47)$ & $222(42)$ & -9 to 1 & -32 to $-22^{b}$ & 17 to $27^{\mathrm{c}}$ \\
\hline Fat $\left(\mathrm{g} \cdot \mathrm{day}^{-1}\right)$ & $48.5(15.4)$ & $41.1(14.5)$ & $49.1(15.8)$ & -2.0 to 0.5 & -9.1 to $-6.6^{\mathrm{b}}$ & 5.9 to $8.4^{\mathrm{c}}$ \\
\hline$\%$ energy intake from protein & $14(4)$ & $14(4)$ & $14(4)$ & -0.1 to 0.7 & -0.2 to 0.6 & -0.3 to 0.5 \\
\hline$\%$ energy intake from $\mathrm{CHO}$ & $58(4)$ & $59(4)$ & $58(4)$ & -1.2 to 0.5 & -0.2 to 1.5 & -1.9 to $-0.2^{\mathrm{C}}$ \\
\hline$\%$ energy intake from fat & $28(5)$ & $27(4)$ & $28(5)$ & -0.7 to 0.9 & -1.7 to $-0.1^{b}$ & 0.2 to $1.8^{\mathrm{c}}$ \\
\hline
\end{tabular}

Values are mean (SD) for $n=16$. $* 95 \%$ confidence interval of the mean absolute difference between the experimental conditions.

CHO, carbohydrate.

${ }^{\text {a }}$ Significant difference between HIIR and CON $(P<0.05)$

${ }^{\mathrm{b}}$ Significant difference between HIIR-ER and CON $(P<0.05)$

${ }^{\mathrm{c}}$ Significant difference between HIIR and HIIR-ER $(P<0.05)$ 
Table 2

Physical activity levels and sedentary time during the intervention day in the high-intensity interval running (HIIR), high-intensity interval running and energy-intake restriction (HIIR-ER) and control (CON) conditions.

\begin{tabular}{|c|c|c|c|c|c|c|}
\hline & HIIR & HIIR-ER & CON & $\begin{array}{c}\text { CON vs. } \\
\text { HIIR } \\
95 \% \text { CI* }^{*}\end{array}$ & $\begin{array}{l}\text { CON vs. } \\
\text { HIIR-ER } \\
\text { 95\% CI* }\end{array}$ & $\begin{array}{c}\text { HIIR-ER vs. } \\
\text { HIIR } \\
\text { 95\% CI* }\end{array}$ \\
\hline Daily wear time (min) & 838 (800 to 877$)$ & 810 (774 to 848$)$ & 808 (772 to 846$)$ & -2 to $9 \%$ & -5 to $6 \%$ & -2 to $9 \%$ \\
\hline Counts per minute & 422 (375 to 476$)$ & 348 (309 to 393) & 295 (261 to 332 ) & 26 to $63 \%^{\mathrm{a}}$ & 4 to $34 \%^{\mathrm{b}}$ & 7 to $38 \%{ }^{\mathrm{c}}$ \\
\hline Sedentary activity (min) & 494 (461 to 530$)$ & 502 (468 to 538$)$ & 521 (486 to 559) & -13 to $4 \%$ & -12 to $5 \%$ & -10 to $8 \%$ \\
\hline Light activity (min) & 248 (215 to 286$)$ & 228 (198 to 263 ) & 224 (194 to 258$)$ & -1 to $24 \%$ & -9 to $14 \%$ & -3 to $22 \%$ \\
\hline Moderate activity (min) & 68 (58 to 80$)$ & 54 (46 to 63$)$ & 50 (43 to 59$)$ & 15 to $59 \%^{a}$ & -10 to $25 \%$ & 8 to $50 \%^{\mathrm{c}}$ \\
\hline Vigorous activity (min) & 14 (9 to 23$)$ & $9(6$ to 15$)$ & $2(1$ to 4$)$ & 260 to $924 \%^{\mathrm{a}}$ & 134 to $565 \%^{\mathrm{b}}$ & -9 to $160 \%$ \\
\hline
\end{tabular}

Values are geometric mean (95\% confidence interval) for $n=16$. Statistical analyses are based on natural log transformed data. $* 95 \%$ confidence interval for the ratio of geometric means.

${ }^{\text {a }}$ Significant difference between HIIR and CON $(P<0.05)$

${ }^{\mathrm{b}}$ Significant difference between HIIR-ER and CON $(P<0.05)$

${ }^{c}$ Significant difference between HIIR and HIIR-ER $(P<0.05)$ 
Table 3 Fasting and postprandial plasma triacylglycerol, non-esterified fatty acids (NEFA) and glucose concentrations in the highintensity interval running (HIIR), high-intensity interval running and energy-intake restriction (HIIR-ER) and control (CON) conditions.

\begin{tabular}{|c|c|c|c|c|c|c|}
\hline & HIIR & HIIR-ER & CON & $\begin{array}{c}\text { CON vs. } \\
\text { HIIR } \\
95 \% \text { CI* } \\
\end{array}$ & $\begin{array}{l}\text { CON vs. } \\
\text { HIIR-ER } \\
95 \% \text { CI* } \\
\end{array}$ & $\begin{array}{c}\text { HIIR-ER vs. } \\
\text { HIIR } \\
\text { 95\% CI* } \\
\end{array}$ \\
\hline \multicolumn{7}{|l|}{ Triacylglycerol } \\
\hline Fasting $\left(\mathrm{mmol} \cdot \mathrm{L}^{-1}\right)$ & $0.74(0.63$ to 0.87$)$ & 0.81 (0.69 to 0.95$)$ & 0.88 (0.75 to 1.03$)$ & -24 to $-7 \%^{a}$ & -17 to $1 \%$ & -17 to $1 \%$ \\
\hline TAUC $\left(\mathrm{mmol} \cdot \mathrm{L}^{-1} 6.5 \mathrm{~h}\right)$ & 7.75 (6.36 to 9.43$)$ & 7.81 (6.41 to 9.51$)$ & 8.58 (7.05 to 10.45$)$ & -16 to $-3 \%^{a}$ & -15 to $-2 \%^{b}$ & -8 to $6 \%$ \\
\hline \multicolumn{7}{|l|}{ NEFA } \\
\hline Fasting $\left(\mathrm{mmol} \cdot \mathrm{L}^{-1}\right)$ & $0.68(0.56$ to 0.81$)$ & 0.70 (0.58 to 0.83$)$ & $0.87(0.72$ to 1.04$)$ & -37 to $-4 \%^{a}$ & -35 to $-1 \%^{b}$ & -21 to $20 \%$ \\
\hline TAUC $\left(\mathrm{mmol} \cdot \mathrm{L}^{-1} 6.5 \mathrm{~h}\right)$ & 2.61 (2.24 to 3.03) & 2.67 (2.30 to 3.11$)$ & 2.51 (2.16 to 2.92) & -6 to $15 \%$ & -4 to $17 \%$ & -12 to $8 \%$ \\
\hline iAUC $\left(\mathrm{mmol} \cdot \mathrm{L}^{-1} 6.5 \mathrm{~h}\right)$ & $-1.79(-2.91$ to -0.38$)$ & $-1.85(-2.95$ to -0.45$)$ & $-3.67(-4.38$ to -2.77$)$ & 14 to $115 \%^{\mathrm{a}}$ & 13 to $113 \%^{\mathrm{b}}$ & -27 to $39 \%$ \\
\hline iAUC $\left(\mathrm{mmol} \cdot \mathrm{L}^{-1} 6.5 \mathrm{~h}\right)$ & 9.75 (7.24 to 12.94$)$ & 6.48 (4.67 to 8.78$)$ & 7.79 (5.70 to 10.45$)$ & -12 to $63 \%$ & -36 to $18 \%$ & 2 to $88 \%{ }^{c}$ \\
\hline
\end{tabular}


TAUC, total area under the concentration versus time curve; iAUC, incremental area under the concentration versus time curve.

${ }^{a}$ Significant difference between HIIR and CON $(P<0.05)$

${ }^{\mathrm{b}}$ Significant difference between HIIR-ER and CON $(P<0.05)$

${ }^{\mathrm{c}}$ Significant difference between HIIR and HIIR-ER $(P<0.05)$ 


\section{Figure 1}

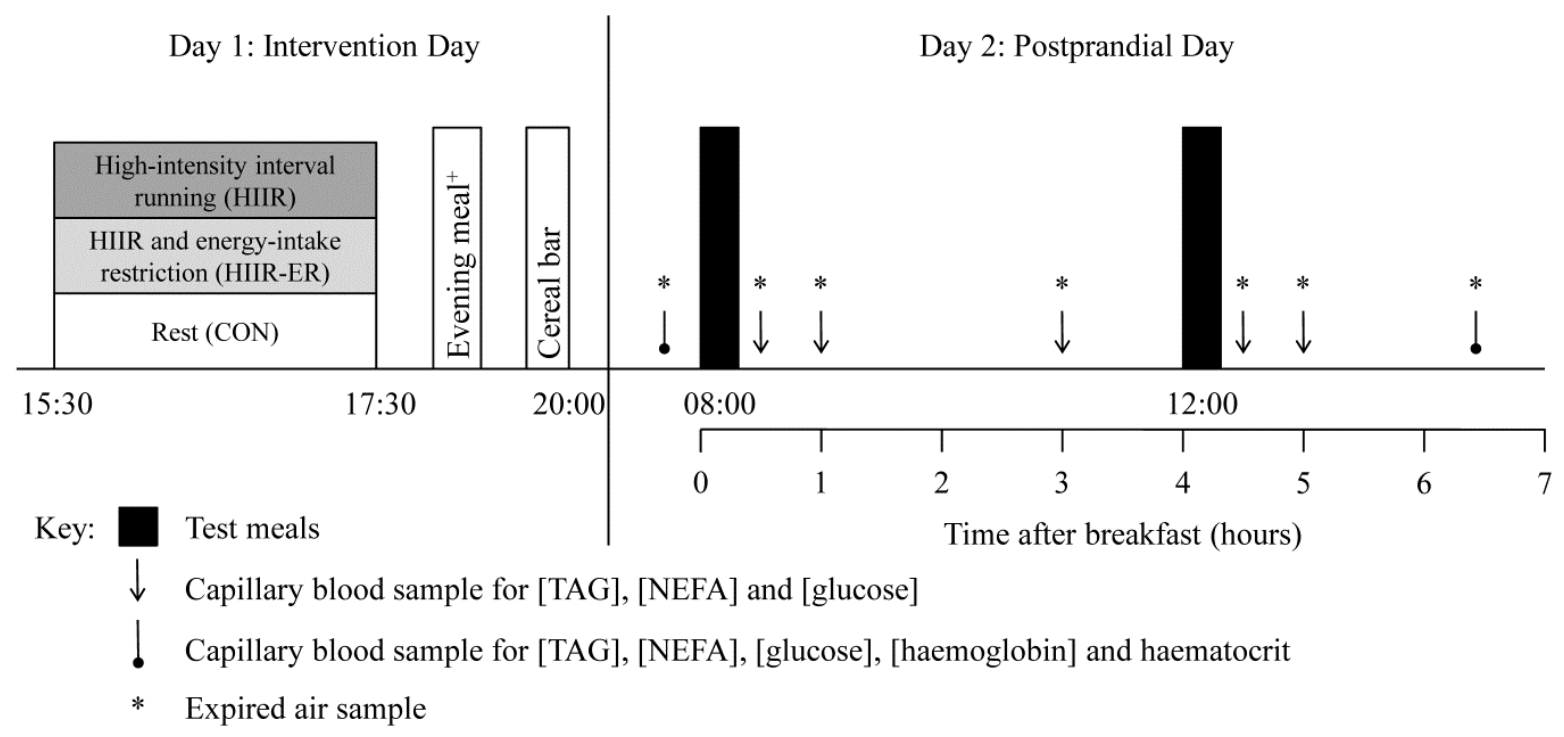


Figure 2

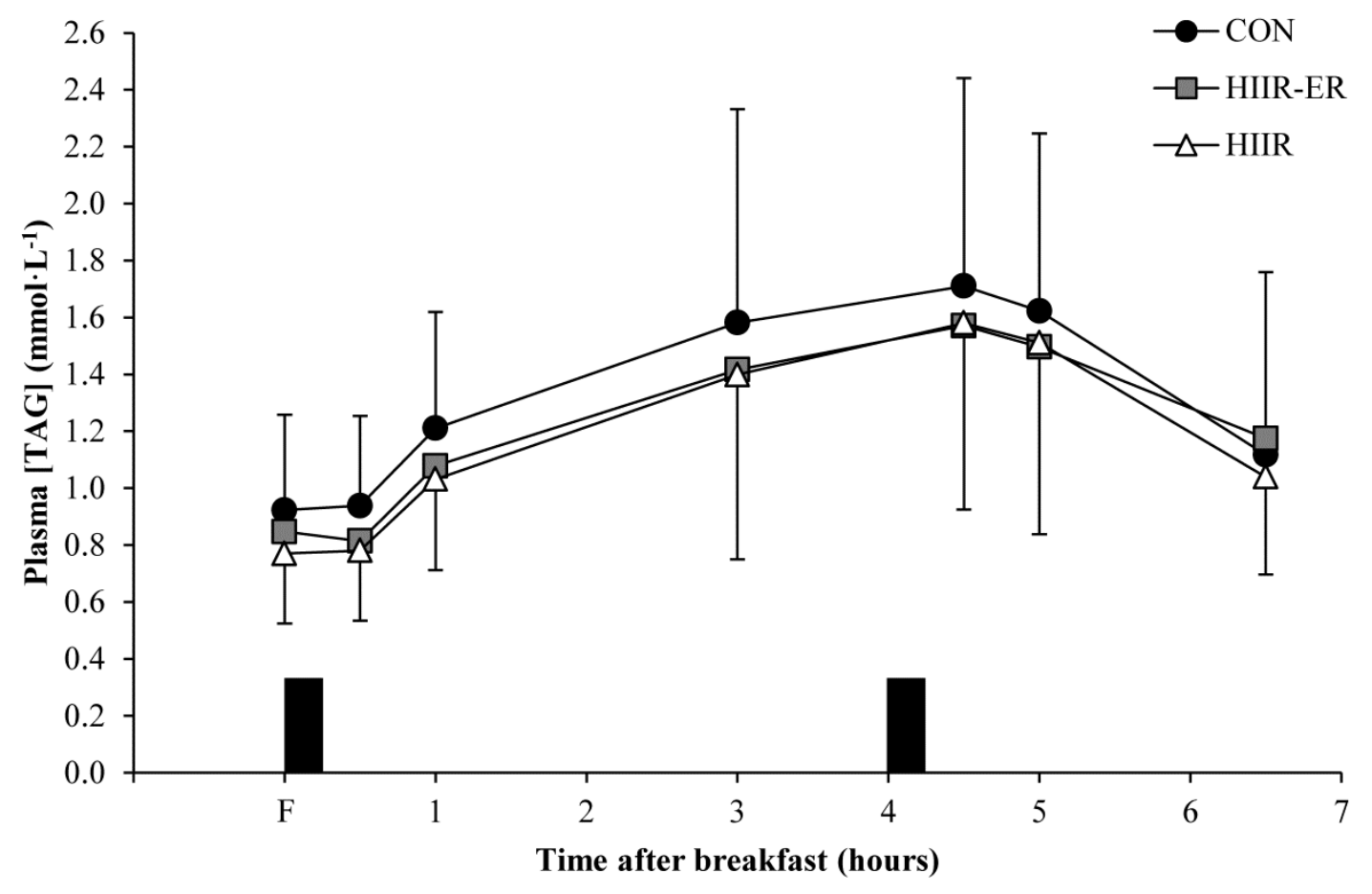


Figure 3

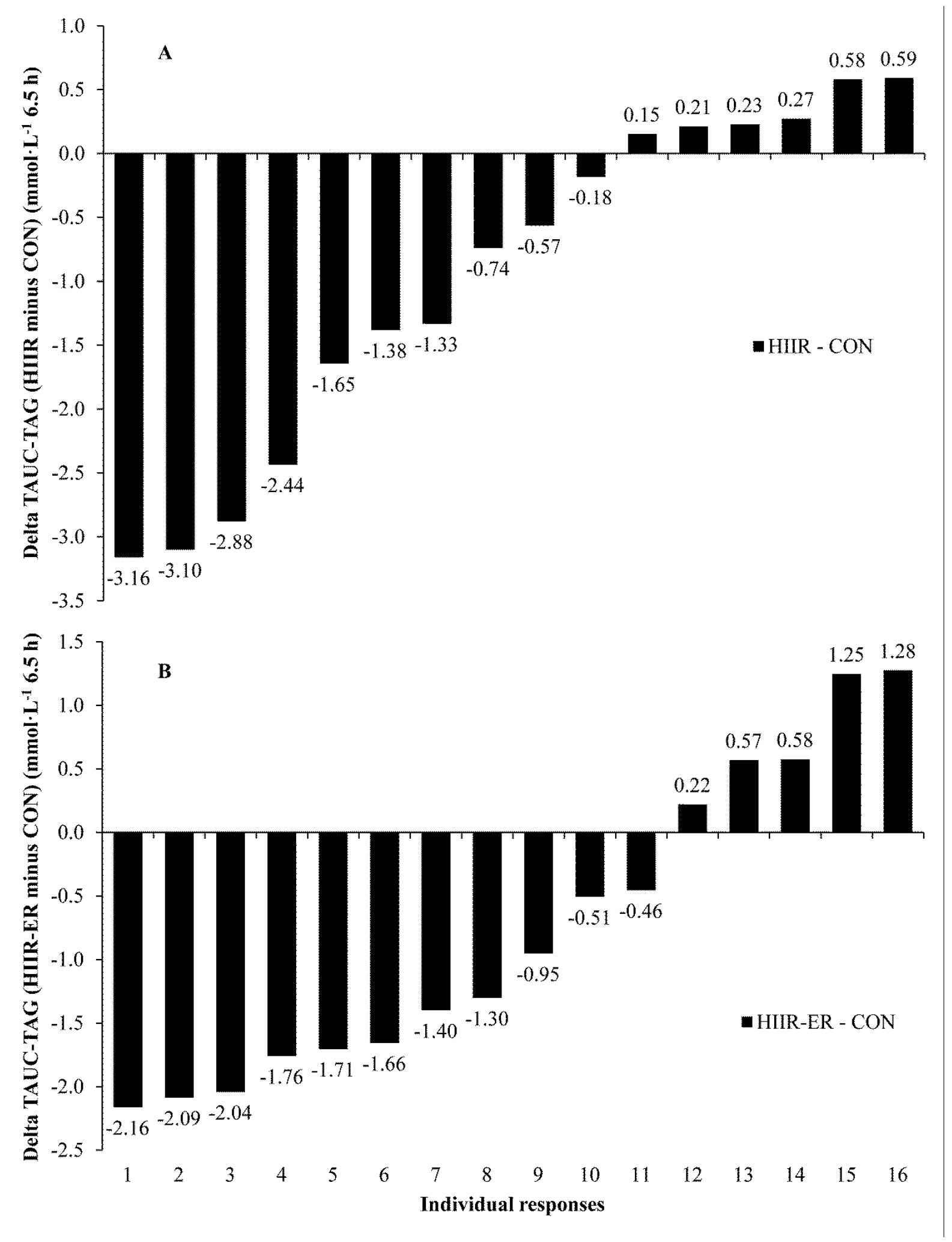

